\title{
Choroidal Drainage
}

\author{
${ }^{1}$ Rajul S Parikh, ${ }^{2}$ Shefali Parikh, ${ }^{1}$ Ravi Thomas \\ ${ }^{1}$ LV Prasad Eye institute, India \\ ${ }^{2}$ Sadhuram Eye Hospital, Charitable Trust and Postgraduate Institute of Ophthalmology, India
}

Ciliochoroidal effusion is collection of fluid in supra-choroidal space, a potential space between sclera and choroid. It is usually seen after intraocular surgery where intraocular or post-operative hypotony is combined with post-operative inflammation. ${ }^{1-7} \mathrm{It}$ may lead to choroidal detachment. In severe choroidal detachment, visual acuity may deteriorate, aqueous fluid formation may reduce and anterior chamber may become shallow due to forward rotation of ciliary body eventually pushing irislens diaphragm forwards.

Choroidal detachment (CD) secondary to choroidal effusion is one of the under diagnosed complications associated with glaucoma filtration surgery. In collaborative initial glaucoma treatment study (CIGTS), 11 percent of patients who underwent Glaucoma filtration surgery had Choroidal detachment. ${ }^{8}$ With the increase in the use of adjunctive antimetabolites in glaucoma filtering surgeries, the resultant prolonged hypotony has led to increased incidence of persistent choroidal detachments. Fortunately, all Choroidal detachments does not require surgical intervention.

The mechanism of formation of serous choroidal effusion is still not clear. One theory suggests that a tear in the ciliary body allows aqueous humor to flow into the suprachoroidal space ${ }^{9}$; recently, experimental models of monkeys with choroidal detachment showed presence of aqueous humor in the suprachoroidal fluid. ${ }^{10}$ Other theory suggests that this fluid is a transudate from across the capillary walls when the intraocular pressure (IOP) is reduced. ${ }^{11}$ Capper and Leopold provided supporting experimental evidence in rabbits, while Moses used physical properties of enucleated eyes to support this theory. ${ }^{12,13}$

The clinical course of CD is variable; spontaneous resolution often occurs as the IOP rises. In most instances, the choroidal detachment is treated medically with mydriatics/ cycloplegics and steroids. ${ }^{2-7}$ Prolonged choroidal detachments can lead to shallow anterior chamber with subsequent peripheral anterior synechiae formation, failure of filtration surgery, cataract formation or hastening of nuclear sclerosis, damage to the corneal endothelium and hypotony maculopathy. In these cases, to restore the normal anatomy and visual function, surgical drainage of persistent choroidal effusions may be required. ${ }^{1-4,14-16}$
If choroidal detachment does not resolve with medical management and persists longer than 1 week even if underlying cause has been addressed, drainage of the suprachoroidal fluid should be considered. However, each case needs individualized assessment. If an improvement is suspected, close monitoring of the patient may be warranted before surgical intervention.

Following are the indication for undertaking prompt surgical intervention:

1. Lenticulo-endothelial touch

2. Progressive corneal edema

3. Failing filtering bleb in an inflamed eye

4. Wound leak with flat anterior chamber

5. Kissing choroidal's for $>48 \mathrm{hrs}$

6. Shallow AC with colarette iridocorneal touch for more than 3 days or peripheral iridocorneal touch for more than 1 week

The surgical drainage of suprachoroidal fluid has been described since the 1960s. Few case series reports good success of this procedure. ${ }^{14-17}$ It is a simple surgical procedure and can be performed by glaucoma specialist and general ophthalmologist who performs trabeculectomy routinely and come across this complication. Unfortunately in India, vitreo-retinal surgeon at quite a few places carries out this procedure. In this article, we attempt to highlight the simplicity of the surgical procedure.

Preoperatively, Indirect ophthalmoscopy and/or B-scan ultrasonography should be done to identify the site of maximum fluid accumulation or choroidal detachment (Figure 1). This should be the site for fluid drainage. Choroidal drainage is done under peribulbar block with 2 percent xylocaine, 0.75 percent Bupivacaine mixed with 1:200,000 epinephrine and hyaluronidase.

\section{Procedure for Choroidal Drainage}

A paracentesis is made with a myringotome directed from the temporal limbus in clear cornea (Figure 2). If a previous paracentesis had been made as part of a previous surgery (glaucoma or combined glaucoma and cataract procedure), efforts are made to re-use the same entry site, since making a new entry in a soft eye is difficult. The anterior chamber is deepened by injecting balanced salt solution (BSS) or air injected with a 30-gauge needle (Figure 3). 

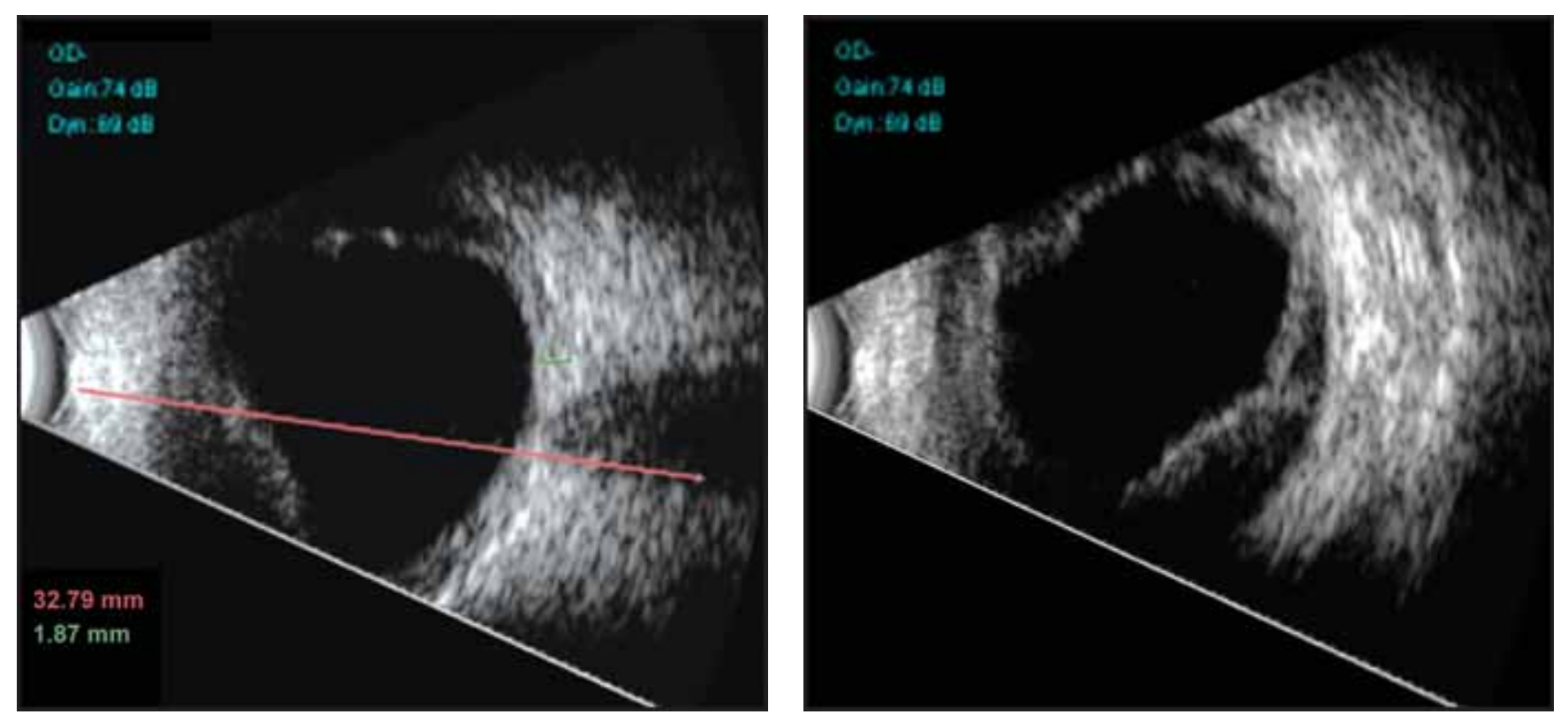

Fig. 1: Ultrasound sonography (B-scan) showing choroidal detachment

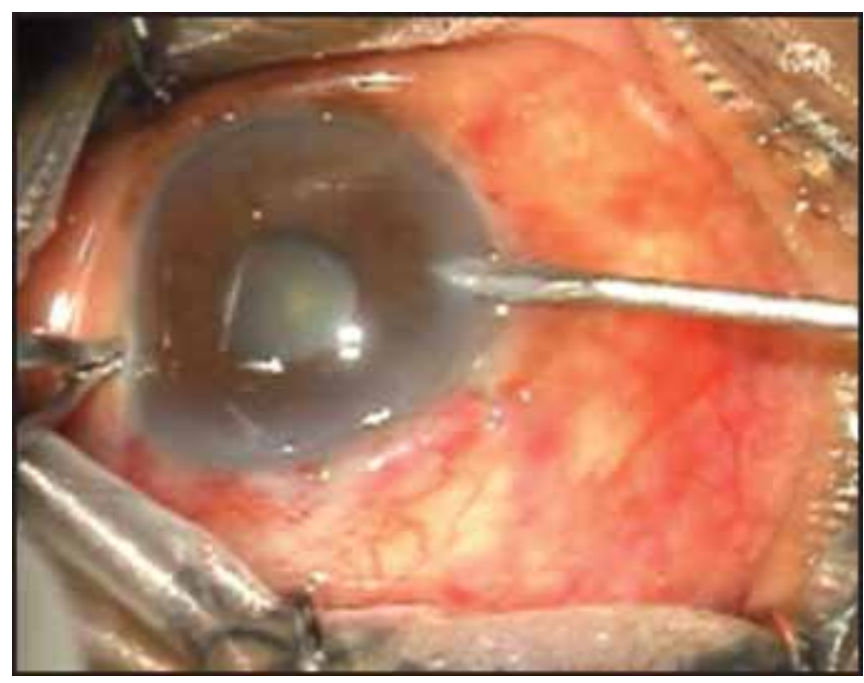

Fig. 2: A myringotomy knife is used to create a beveled incision in the upper temporal quadrant

A circumferential conjunctival incision is made $4 \mathrm{~mm}$ from the limbus in the inferior temporal quadrant/ the site of maximum fluid localization. Using calipers, the site for the choroidal drainage procedure is marked (Figure 4). The center of the incision should be at least $4 \mathrm{~mm}$ from the limbus in phakic patients. While grasping the globe firmly and exposing the quadrant to be drained, a 2 to $3 \mathrm{~mm}$ long, radial incision (sclerotomy) is made at about 4 to $5 \mathrm{~mm}$ from the surgical limbus in the selected quadrant with a 15 no. Bard Parker blade (Figure 5). A more posterior incision should better be avoided

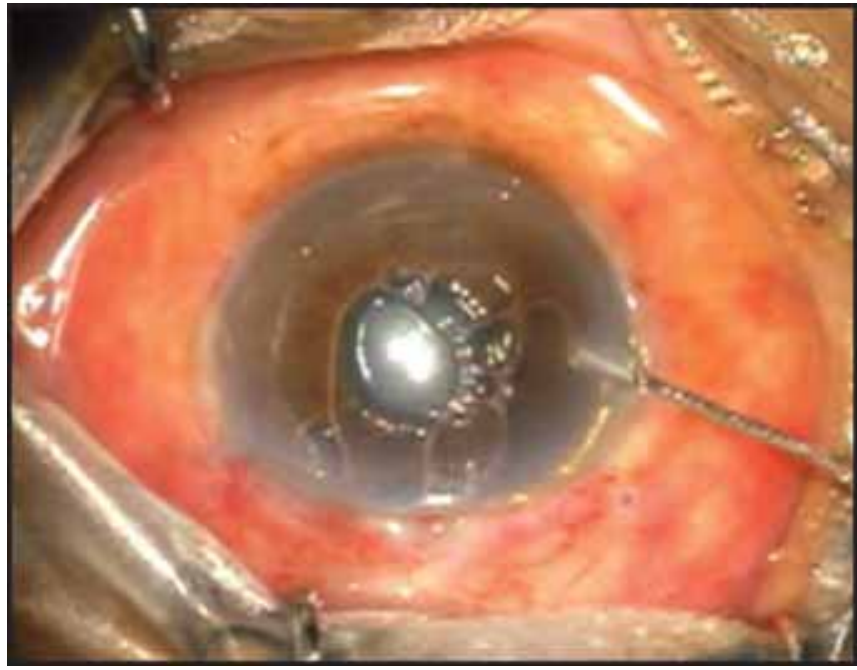

Fig. 3: Air is injected from paracentesis site in to anterior chamber (AC) to form the AC. Balanced Salt Solution (BSS) also can be used instead of air

to minimize the risk of inadvertent retinal perforation and vitreous loss. The edge of the incision should be cauterized with wet field cautery, so that the edge of wound gets retracted (Figure 6). While skillfully maneuvering the blade along the length of the incision, incision is gradually deepened. As the suprachoroidal space is reached, a spontaneous gush of clear yellow serous fluid is seen (Figure 7). As the spontaneous flow slows down, gentle pressure with a blunt instrument like iris repositor a few millimeters around the sclerotomy site helps in draining residual fluid (Figures 8 and 9). A full-length 


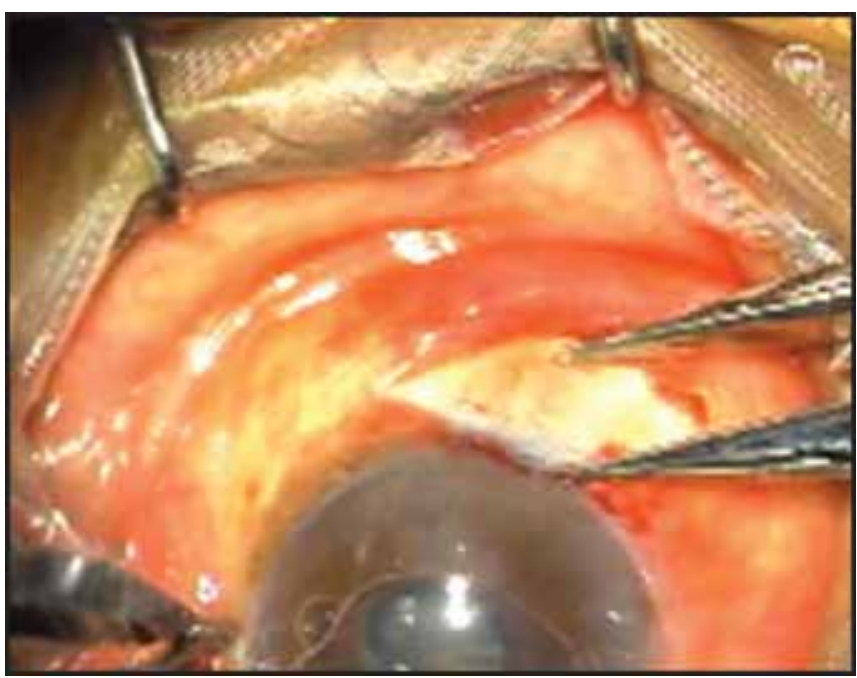

Fig. 4: Using caliper, site is marked for the choroidal drainage procedure. The center of the incision should be at least $4 \mathrm{~mm}$ from limbus in phakic patient

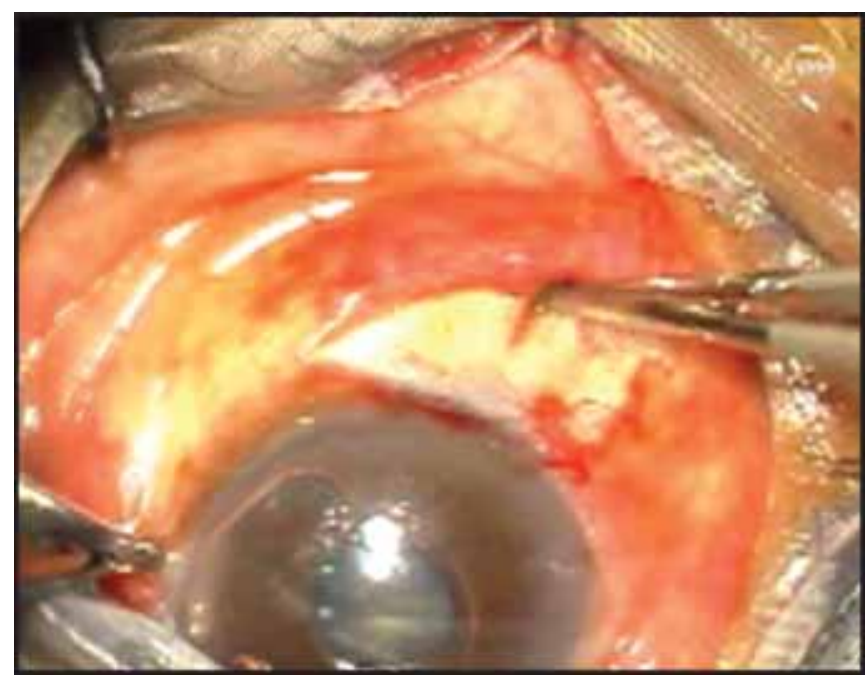

Fig. 6: Edge is cauterized with wet field cautery

cyclodialysis spatula can be inserted under the sclera to drain the fluid from the loculated pockets. However, it should be avoided to reduce the chances of damage to perforating branches of anterior ciliary artery or one of the long posterior ciliary arteries. Care should be taken to fill the anterior chamber with air or BSS to prevent any endothelial damage (Figures 10 and 11). At the end, air bubble should be left in the anterior chamber. The sclerostomy site should be left open, and may be cauterized a little more to ensure further drainage of suprachoroidal fluid in the post-operative period. Conjunctiva

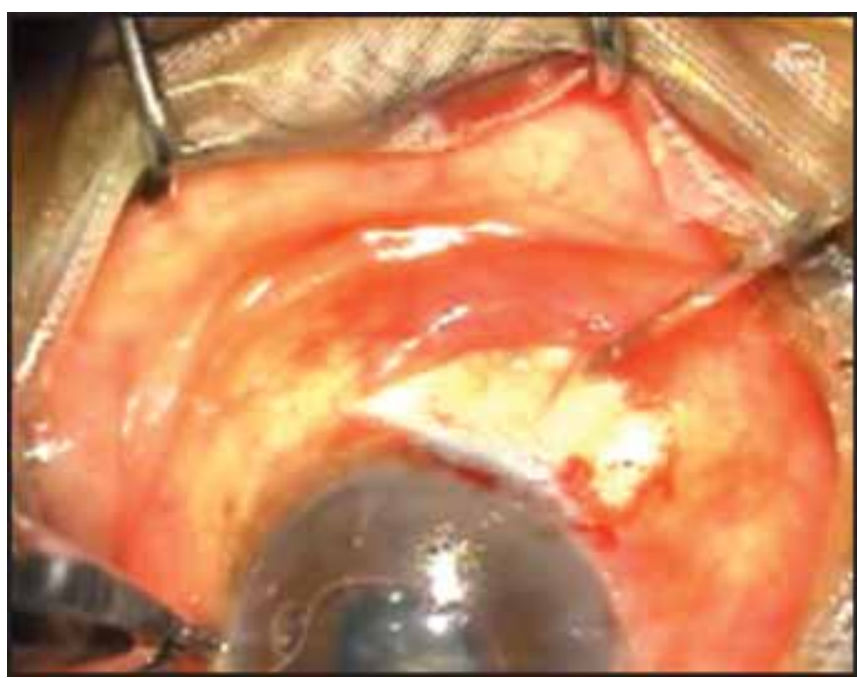

Fig. 5: Using 15 no. Bard Parker blade, 2-3 mm long radial incision is made from the center of the site

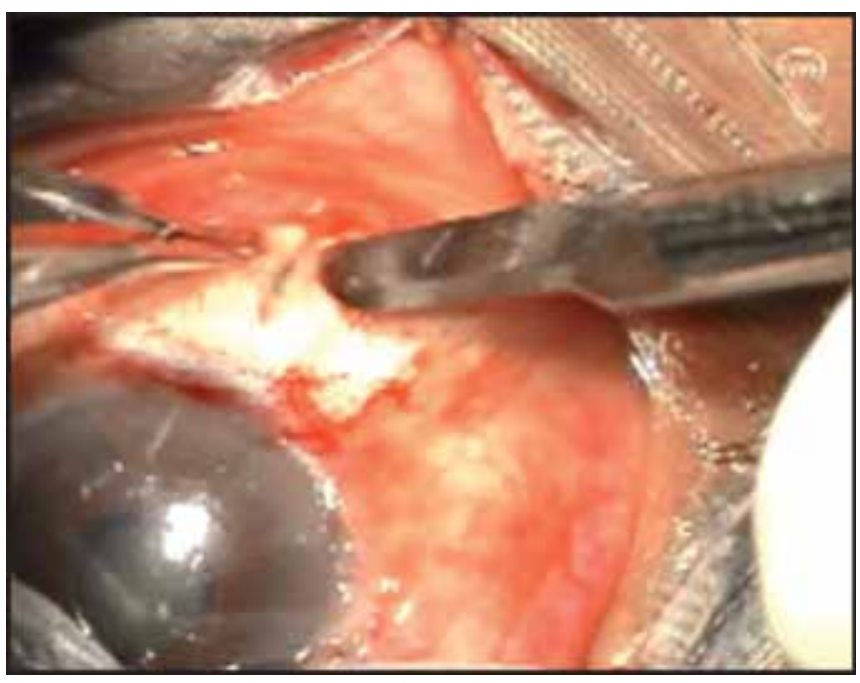

Fig. 7: The wound is slowly deepened till the straw coloured fluid escapes from the wound. The wound has to be deepened very slowly to prevent retinal break

is sutured in a continuous mattress fashion with 8-0 vicryl sutures. Similarly the procedure can be repeated in other quadrants if indicated. This procedure can be repeated for all 4 quadrants in the same or separate sittings.

Postoperatively, a course of topical antibiotics, topical steroids and cycloplegics are advised.

To conclude, the surgical procedure for choroidal drainage procedure is safe, effective and can be performed easily whenever indicated. 

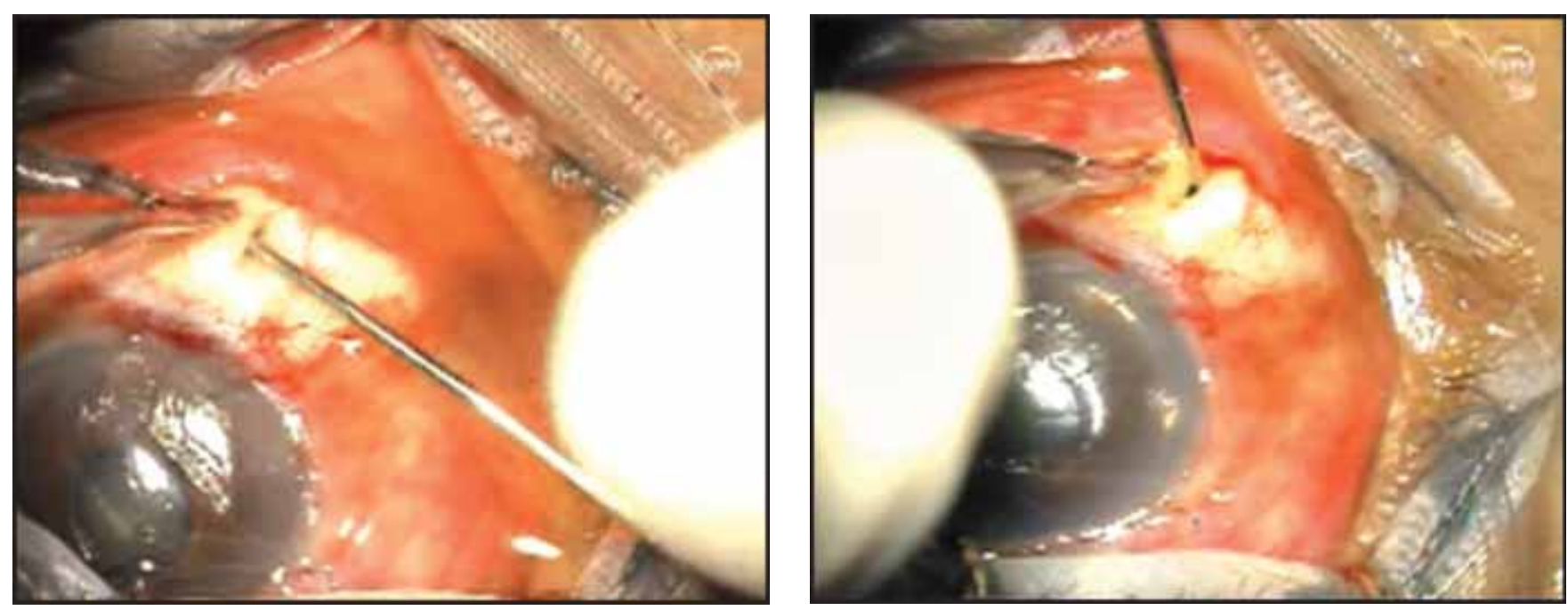

Figs 8 and 9: Cyclodialysis spatula is inserted between sclera and choroid to drain fluid

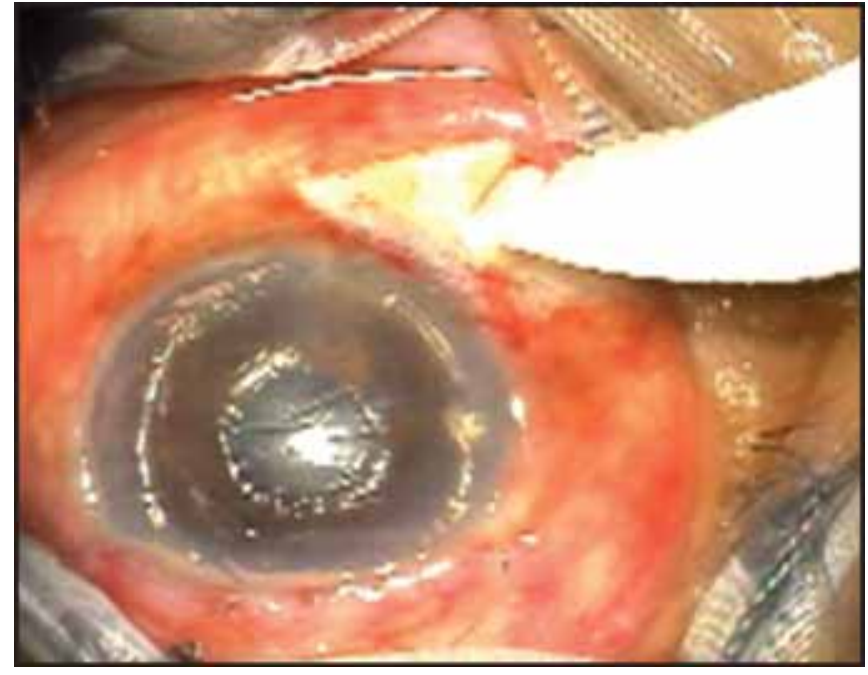

Fig. 10: Surgical wound is always kept open

\section{REFERENCES}

1. Bellows AR, Chylack LT Jr, Hutchinson BT. Choroidal detachment. Clinical manifestation, therapy and mechanism of formation. Ophthalmology. 1981;88:1107-15.

2. Saxena RC, Kumar K. Choroidal detachment (a clinicoaetiopathological study). Indian J Ophthalmol. 1982;31:23841.

3. Brubaker RF, Pederson JE. Ciliochoroidal detachment. Surv Ophthalmol 1983;27:281-9.

4. Berke SJ, Bellows AR, Shingleton BJ, et al. Chronic and recurrent choroidal detachment after glaucoma filtering surgery. Ophthalmology 1987;94:154-62.

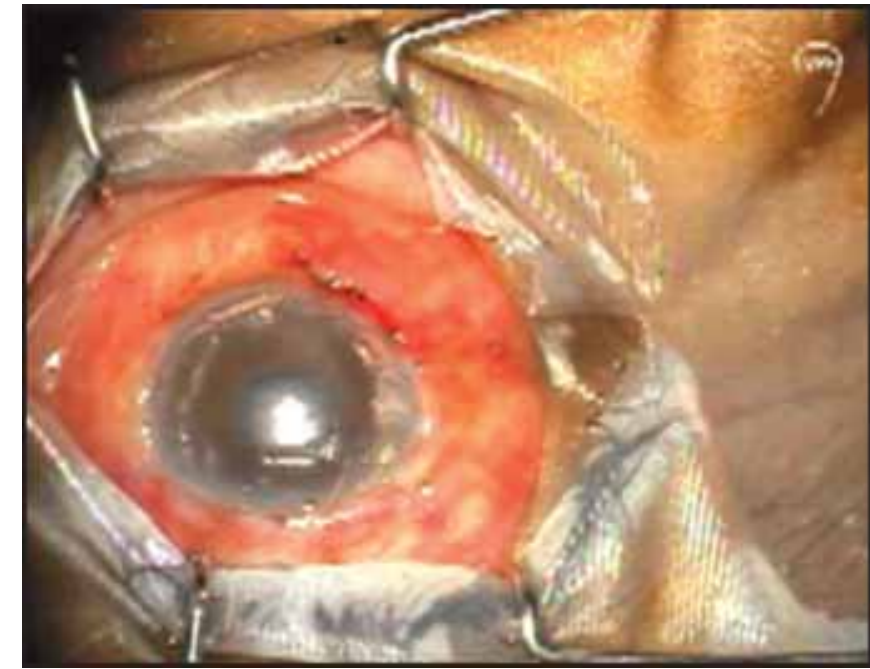

Fig. 11: The conjunctiva is sutured with 8-0 vicryl suture $A C$ is filled with air

5. Burney EN, Quigley HA, Robin AL. Hypotony and choroidal detachment as late complications of trabeculectomy. Am J Ophthalmol 1987;103:685-8.

6. Schubert HD. Postsurgical hypotony: relationship to fistulization, inflammation, chorioretinal lesions, and the vitreous. Surv Ophthalmol 1996;41:97-125.

7. Popovic V. Early choroidal detachment after trabeculectomy. Acta Ophthalmol Scand 1998;76:367-71.

8. Jampel HD, Musch DC, Gillespie BW, Lichter PR, Wright MM, Guire KE; Collaborative Initial Glaucoma Treatment Study Group. Perioperative complications of trabeculectomy in the collaborative initial glaucoma treatment study (CIGTS). Am J Ophthalmol. 2005;140:16-22. 
9. Fuchs E. Ablosung der Aderhaut nach staaroperation. Albrecht von Graefes Arch Ophthalmol. 1900;51:199-224.

10. Pederson JE, Gaasterland DE, MacLellan HM. Experimental ciliochoroidal detachment effect on intraocular pressure and aqueous humor flow. Arch Ophthalmol 1979;97:536-41.

11. Meller J. Uber postoperative und spontane choriodealabhebung. Albrecht von Graefes Arch Ophthalmol 1912;80:170-205.

12. Hudson AC. Serous detachment of the choroids and ciliary body as an accomplishment of perforating lesions of the eyeball. Royal London Ophthalmic Hosp Rep. 1914;19:301-10.

13. Moses RA. Detachment of ciliary body-anatomical and physical considerations. Investigate Ophthalmol 1965;4:03541.

14. Cotlier E. Aphakic flat anterior chamber. III. Effect of inflation of the anterior chamber and drainage of choroidal detachment. Arch Ophthalmol 1972;88:16-21.

15. Krishnan MM, Baskaran RK. Management of postoperative choroidal detachment. Indian J Ophthalmol. 1985;33:217-20.

16. Liebmann JM, Sokol J, Ritch R. Management of chronic hypotony after glaucoma filtration surgery. J Glaucoma 1996;5:210-20.
17. WuDunn D, Ryser D, Cantor LB. Surgical Drainage of Choroidal Effusions Following Glaucoma Surgery. J Glaucoma 2005; 14:103-08.

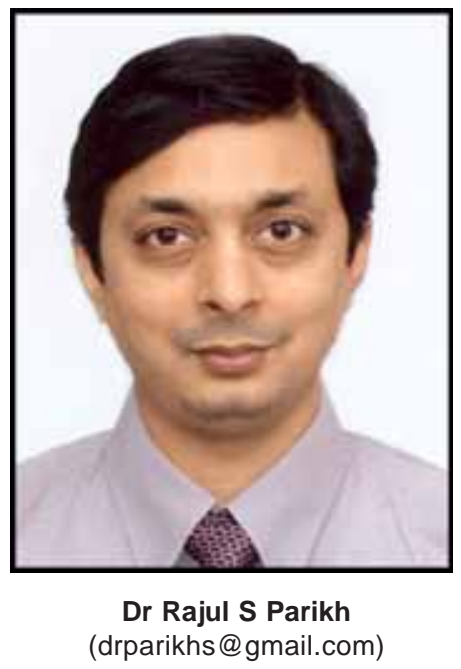

"The way to gain a good reputation is to endeavor to be what you desire to appear" 